\title{
Design of Experiments:
}

New research design could lead to better crops

As the world's population continues to grow, new ways to ensure the future Many crops undergo genetic transformation - a process where new genes are introduced into a plant cell, Which are then grown into hasten crop improvement is to optimise the transformation process. With this aim, Uyen Cao Chu of Corteva Agriscience ${ }^{T M}$, Johnston, lowa USA, has used an approach called Design of Experiments to optimise the transformation process in maize. This method allows many variables to be of time.

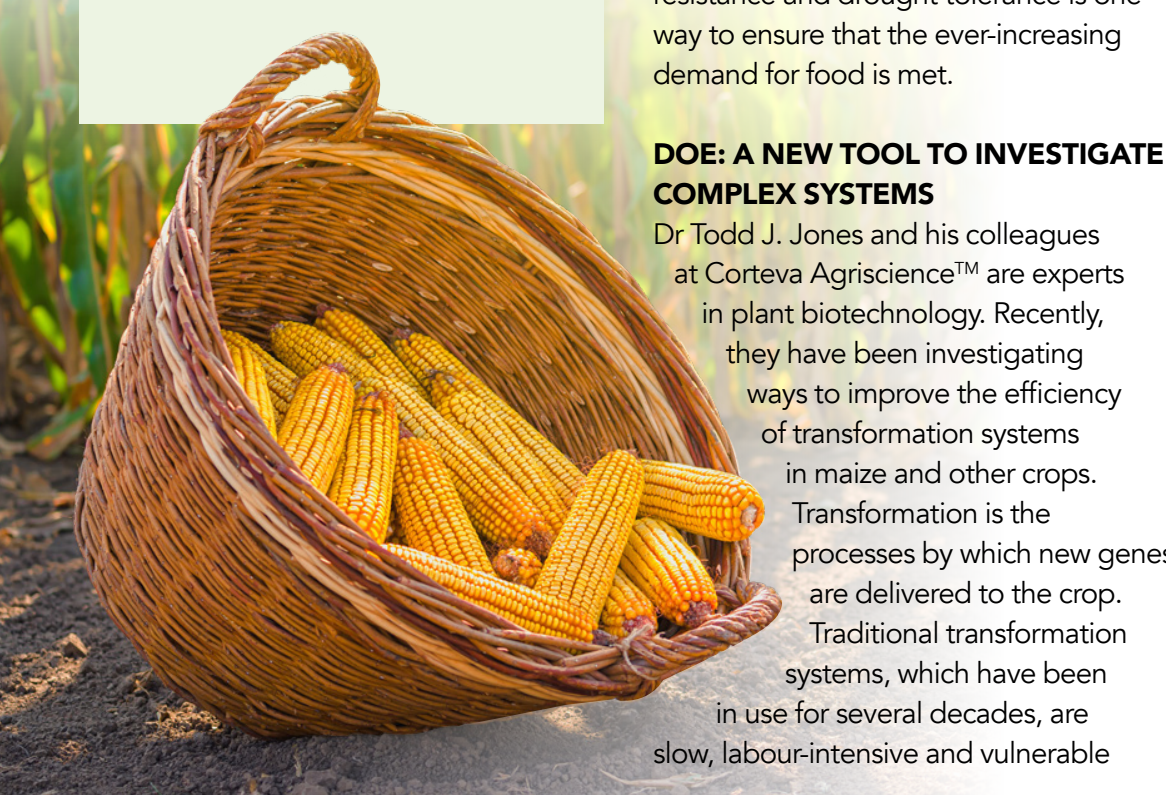

to human error. The whole process, from the development of a mass of cells called callus (the source of the cells that eventually become new plants) to the growth of plantlets, takes around 10 15 weeks.

In 2016, Dr Jones was a part of a feam that identified two genes, called Babyboon (BBM) and Wuschel2 (Wransf), increase the efficiency of different stages of the transformation process and broaden the range of germplasm that can be transformed. This new system, using BBM and WUS2, has since been applied to other cereal crops and has proven to be a rapid, universal transformation system.

Despite these improvements, however, few other previous studies have attempted to improve efficiency by increasing the quality of the transformation process. To explore which factors might result in a higher quality process, Ms Chu used an approach called of statistical metholology. Da is a form multiple factors in a complex system to be tested simultaneously: basically it find cause-and-effect relationships within a system. DoE allows systems to be tested to an extent that would be prohibitively time-consuming and/or expensive with traditional one-factor-at-a-time methods.

FROM PETRI DISH TO GREENHOUSE Ms Chu used DoE to investigate a total of ten different factors that affect plant regeneration and the development of healthy roots. These include the form and concentration of nitrogen, an essential plant nutrient needed for protein synthesis, and the type and relative concentrations of plant growth The aim of the study was to identify

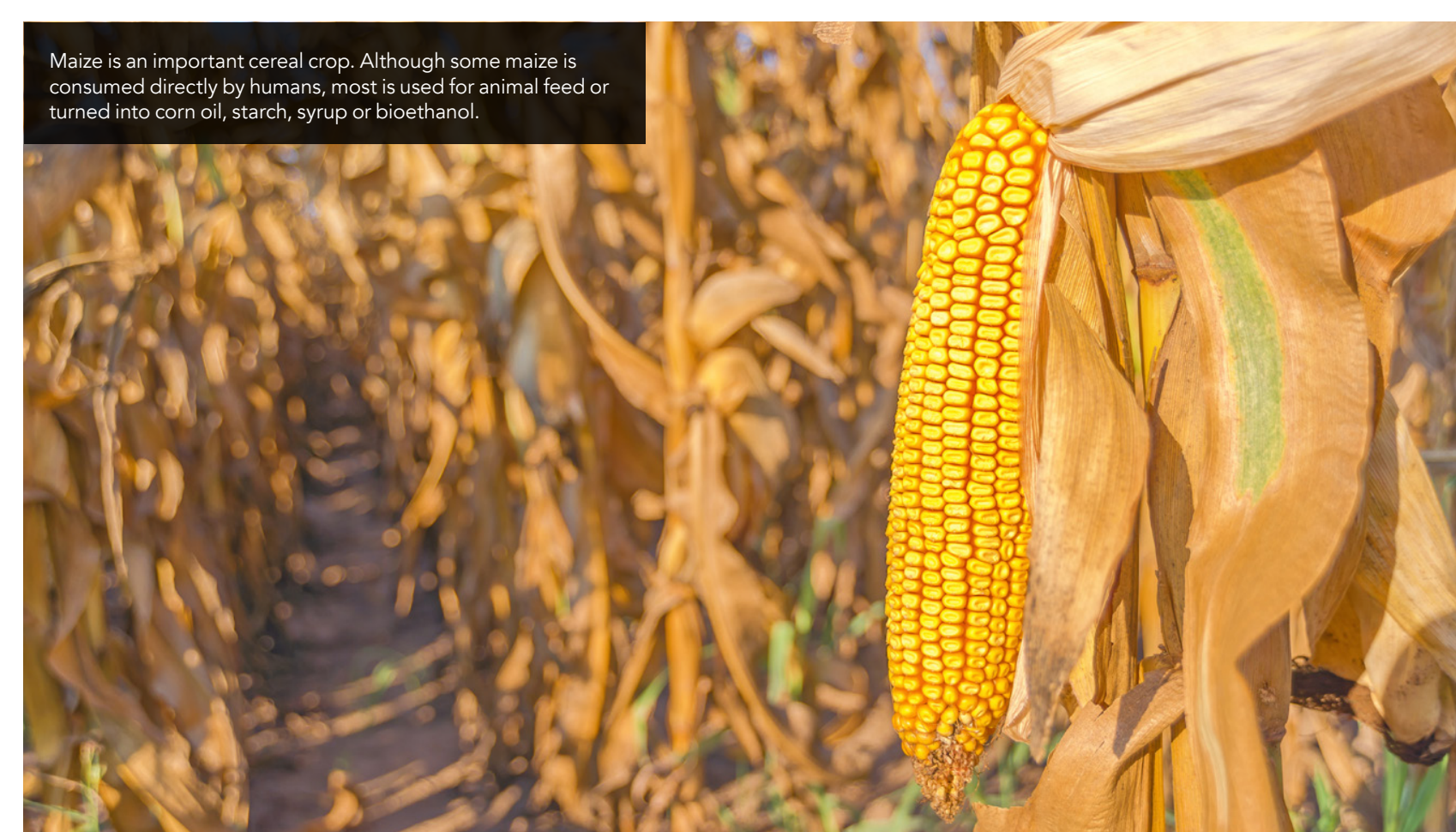

how each of these factors should be applied in the transformation process or example, how much of process should be given to the plants - to optimise the quality of the plant at evey stage of growth.

The team used a well-established transformation process, which begins with isolating maize embryos and infecting them with a bacterium called Agrobacterium tumefaciens, which can efficiently deliver new genes into maize. stimulated by the expression of WUS2 thut and BBM and the somatic embryos plants have developed roots, they are moved to a greenhouse and transplanted into soil. There, the plants grow to maturity. After pollination, the plants produce seed (the kernels on a corn cob most people envisage when they think of maize), which is harvested after around three to four months. The team measured the responses of the somatic embryos to the various factors at all stages of the transformation process.

A BETTER, HIGHER QUALITY WAY TO GROW MAIZE Ms Chu found that many of the ten factors studied had a significant impact from the very first ematon process, fom the very first embryonic stages to to the greenhouse. In particular, the two macronutrients (nutrients that are needed in large quantities), nitrate and the ratio of ammonium to potassium, proved to be important in every response
measured. Light and PGRs were also

significant, but not in every response. In early development, low light intensity produced the best results. Later on, rooted shoots needed a higher light intensity for optimum development.

Potentially, DoE is an extremely useful tool for investigating complex systems.

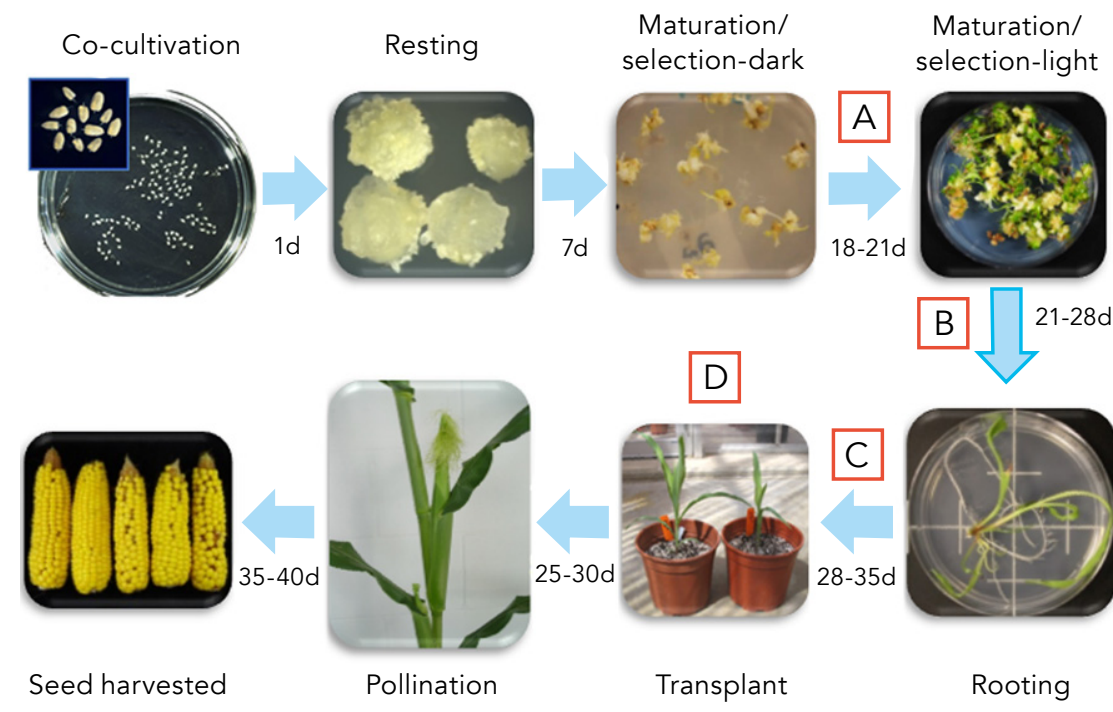

Fig 1. QuickCrop process takes from 117 to 138 days from Agro infection to seed harvest.
The process starts with isolating immature zygotic embryos and infecting with Agrobacterium. Distinct, individual somatic embryms form directly from the scutellem of the eygotic embrryo and
without callus formation. Somatic embryos grow under maturation/selection for $21-25$ days and without callus formation. Somatic embryos grow under maturation/solection for $21-25$ days and
are directly germinated into plants starting 1 month after infection. Following germination and an additional rooting stete, plants sere sent to greenhouses. Transplanted To plantlets are grown to
maturity, pollinated and seed is harvested after approximately $3-4$ months. Letters A, B, C and D 


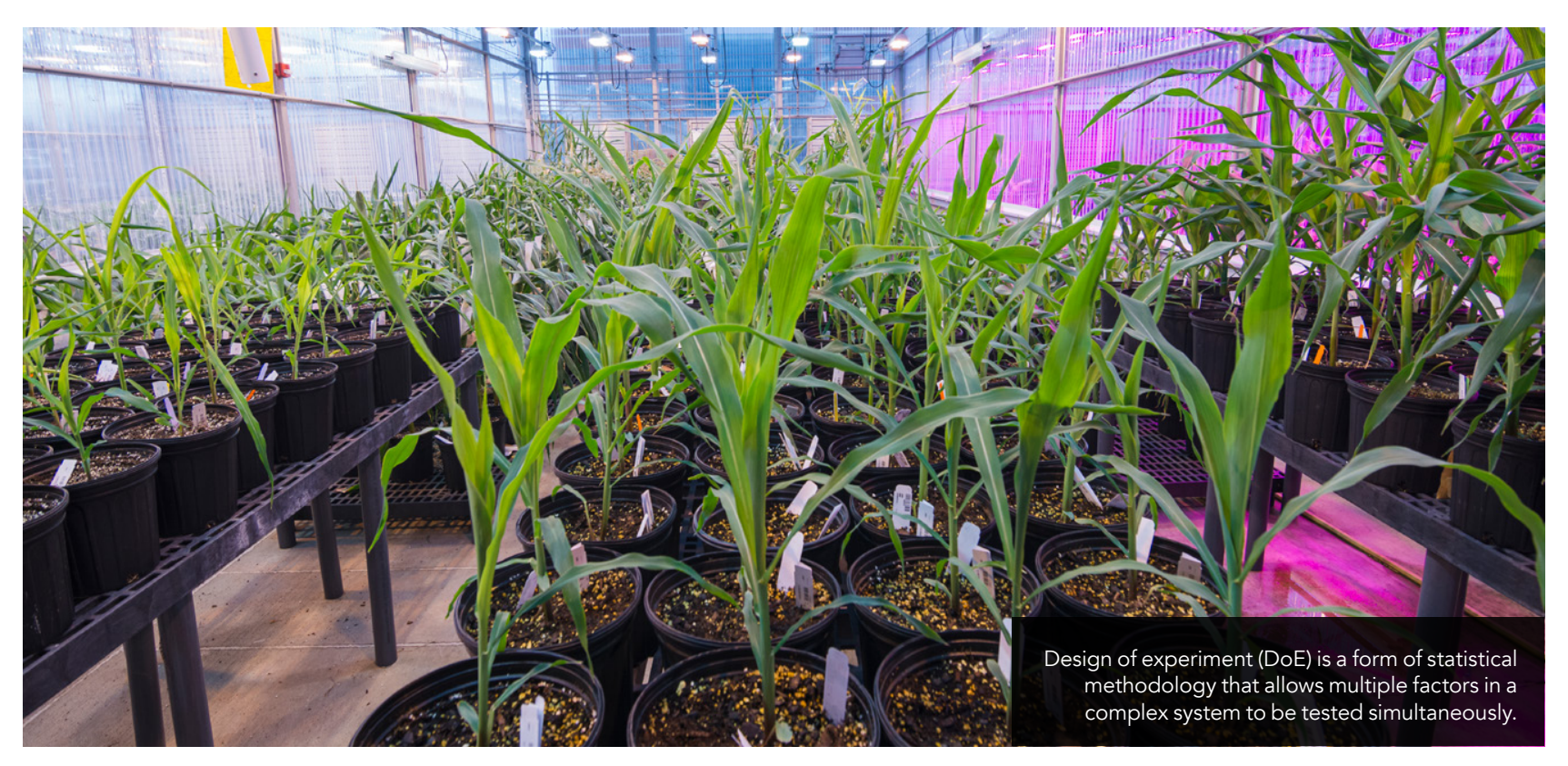

to identify nutrient combinations questin problems the transformation process. It was also possible to identify the interactions between these nutrients and other factors such as light and PGRs.

During early embryo development, the ratio of ammonium to potassium was found to be crucial to the development of the plant. The most effective ratio for these two macronutrients turned out to be 1:1. This resulted in small, single and wellformed shoots that were strong and turgid. However, this changed later in the development of the plants, when more potassim is needed relative to anmolium. This allows the developm leads to healthier, stronger plants once the maize has been transfered to the

The growth process, from transformed tissue to plantlets in the greenhouse, might be best encouraged by a dynamic system.

greenhouse. These results suggest that the growth process, from transformed tissue to plantlets in the greenhouse, might be best encouraged by a dynam system, where factors are varied in order to maintain optimal condition at every stage.

OPTIMISING SYSTEMS WITH DOE Ms Chu found that, by using the optimised conditions identified in this study, the number of plants sent to the with the current process. Genetic-level results also suggest that the regener
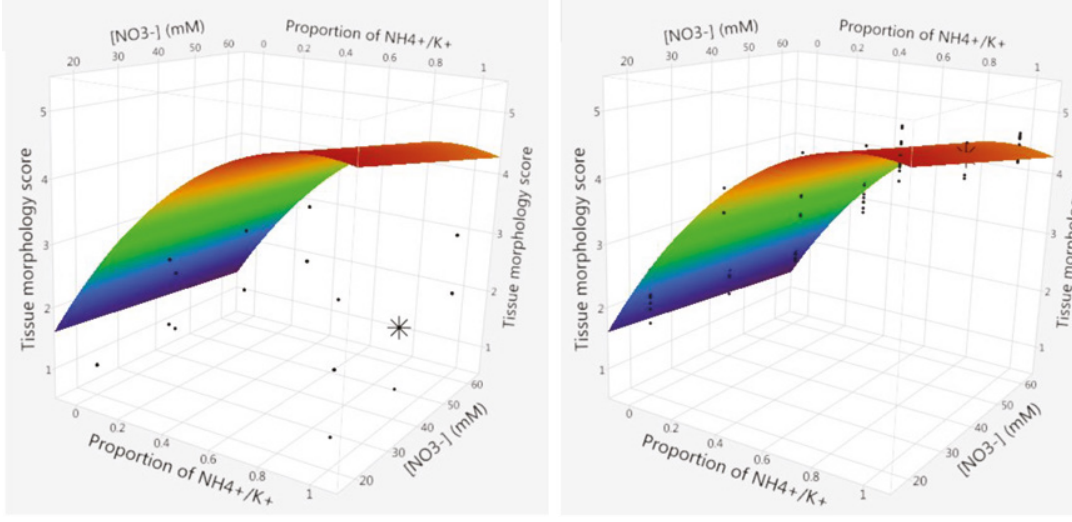

plants grown under these conditions are likely to be more robust.

The valuable results gained in this research could only be achieved by using DoE. The traditional, one-factor-at-a-time experimental approach would have been far slower, requiring so many separate experiments that it is very unlikely to have been attempted. The chances of optimising a complex system, such as these methods woth process, using . researchers to investigate multiple complex variables, and the interactions between those variables, in a greatly reduced time frame.

Potentially, DoE is an extremely useful tool for investigating complex systems, are typically easily manipulated in controlled conditions. The work by Ms Chu has clearly illustrated this potential, and proven DoE to be an effective way to optimise the growth of transformed maize. If the challenges of feeding an ever-increasing population are to be met, studies like this could prove cruci in ensuring the
food supply. particularly in vitro, as these experiments

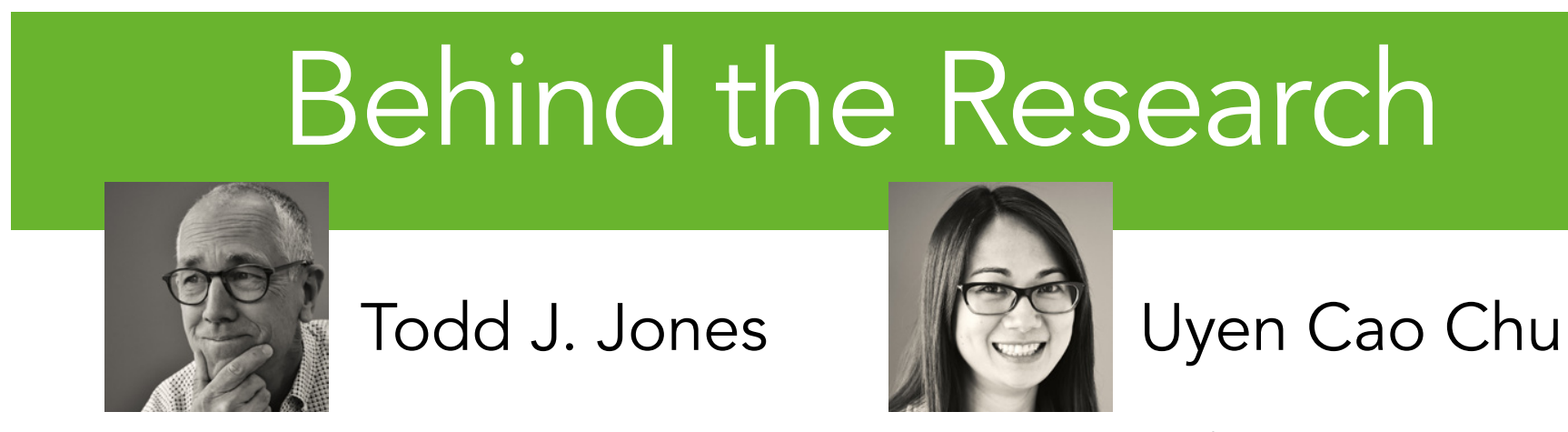

E: todd.j.jones@corteva.com
T: +1 $9193480201 \quad$ T: +15155356251

E: uyen.chu@corteva.com
T: $+15158685488 \quad$ T: +1 5155356196

Research Objectives

Dr Todd J. Jones manages teams responsible for all aspects of crop transformation and genome editing. This includes the W Jer morphogenic genes to enhance transformation, and the discovery of new microbes for plant transformation. This research also satisfied the creative component requirement for the completion of Uyen Cao Chu's Master's degree at lowa State University.

\section{Detail}

8305 NW 62 nd Ave Johnston, IA 50131

Bio

Uyen Cao Chu is a Senior Research Associate in the Crop Genome Engineering group at Corteva
Agriscience $T^{T}$, Johnston, IA. She received her Master's Degree in Seed Technology and Business Management from lowa State University. She

has broad experience in plant transformation and genome editing of various crop species and has a keen to improve the transformation and editing processes.

Todd J. Jones is the Research Director for the Crop Genom Engineering group at Corteva Agriscience ${ }^{\mathrm{TM}}$, located in
Johnston, IA. He received his PhD from UC-Davis and has over 30 years of experience working in plan on plant tissue with an emphasis transformation and CRISPR-Cas genome editing.

Collaborators - Jeffrey Adelberg - Keith Lowe

\section{References}

Chu, U.C., et al. (2019). Use of DoE methodology to optimise the regeneration of high-quality, single-copy ( Deveric Zea mays L. (maize) plants. In Vitro Cellubr org/10.1007/s11627-019-10002-w

Lowe, K, et al. (2018). Rapid genotype "independent" Zea mays L. (maize) transformation via direct somatic embryogenesis. In Vitro Cellular \& Developmental Biology - Plant 54, 240-252. doi.org/10.1007/s11627-0189905-2

Niedz, RP \& Evens, TJ. (2016). Design of experiments (DoE) - history, concepts and relevance to in vitro culture. In Vitro Cellular \& Developmental Biology - Plant. 52, 547562. doi:10.1007.11627-016-9786-1

E CORTEVA

\section{Personal Response}

\section{To what extent could the knowledge gained} in this study be applied to other crops?

II DoE methodology can, and indeed has, been applied to tissue culture and transformation of other
crops. It is exceptionally useful for optimising plant tissu culture conditions where you need to examine multiple variables and understand he interactions between those typical one-factor-at-a-time experiments, it is impossible to determine interaction affects. In our lab, we routinely use DoE methodology to optimise tissue culture
conditions for any crop that we work on. 